\title{
Sub-acute Sclerosing Pan-encephalitis in a 5 Years Old Child: A Response to Intravenous Immunoglobulin and Steroids
}

\author{
Al Shehhi. Wafaa ${ }^{1}$, Alkindi. Hanan ${ }^{2}$, Macki. Nabil ${ }^{1}$, AL Hashami. Hilal ${ }^{3, *}$ \\ ${ }^{1}$ Pediatric Neurology, Royal Hospital, Muscat, Oman \\ ${ }^{2}$ Virology Department, National Public health lab, Muscat, Oman \\ ${ }^{3}$ Pediatric Infectious Diseases, Royal Hospital, Muscat, Oman \\ *Corresponding author: hashamihs@gmail.com
}

\begin{abstract}
A five years old Yemeni boy presented with a 5 months history of progressive neurological deterioration in picture of progressive cognitive decline, seizures and hyperkinetic movements. Patient came from an area with political instability and low socio-economic status. There was history suggestive of measles at 2 years of age. He never received measles vaccination. Diagnosis of sub acute sclerosing pan encephalitis (SSPE) was made based on clinical symptoms, serum and CSF findings of high measles IgG, neuroradiological findings as well as neurophysiological findings. We report this case to highlight the role of intravenous immunoglobulin and steroid in improving his symptoms.
\end{abstract}

Keywords: subacute sclerosing pan encephalitis, children, intravenous immunoglobulin and steroids

Cite This Article: Al Shehhi. Wafaa, Alkindi. Hanan, Macki. Nabil, and AL Hashami. Hilal, "Sub-acute Sclerosing Pan-encephalitis in a 5 Years Old Child: A Response to Intravenous Immunoglobulin and Steroids." American Journal of Medical Case Reports, vol. 5, no. 8 (2017): 226-228. doi: 10.12691/ajmcr-5-8-6.

\section{Introduction}

Subacute sclerosing pan encephalitis (SSPE) is a devastating rare brain disease of young children that should be in the differential diagnosis in children presented with history of infection with measles in the early childhood especially if they come from low socioeconomic area and not receiving proper vaccination. Defective measles virus causes SSPE, after a latent period of 6-8 years [1,2,3]. Affected individuals present with poor school performance and progressive intellectual deterioration, personality changes and behavior abnormalities. This is followed by steady motor decline, myoclonus, focal paralysis, seizures, autonomic failure, and rigidity, finally leading to death with akinatic mutism $[1,2,3]$.

Diagnosis may not be clear initially but as the disease progresses, the diagnosis can be based on several criteria including clinical symptoms, EEG and brain MRI findings as well as measurement of measles antibodies in serum and CSF. The management is based on relieving symptoms and prolonging life span. Immune-modulating agents and anti-viral medications are found to play a role in management of this disease.

\section{Case Report}

A previously healthy 5 years old Yemeni boy, presented with 5 months history of progressive neurological deterioration, the family reported behavioral changes and memory problems after history of mild head trauma that worsening with time to include generalized tonic clonic seizures. He had history of progressive neurological deterioration. No history of fever reported since the onset.

When child first seen in our hospital, he was encephalopathic, choreoathetoid movements and frequent myoclonus involving the head and upper extremities complicated by occasional generalized tonic clonic seizures. Oro-buccal dyskinesia, axial and appendicual hypotonia with brisk reflexes and gereralized weakness was also observed.

Lumbar puncture was performed and CSF sample biochemistry was within normal limits, grams satin,AFB, bacterial culture and TB culture were all negative. CSF molecular test was done using multiplex PCR test and was negative for the common viral agents causing encephalitis. Measles PCR test was also done on the CSF sample and was negative but quantitative antibody serology was positive at level of $12100 \mathrm{mIU} / \mathrm{ml}$ with CSF to serum measles antibody to albumin ratio of $>10$ confirming intrathecal production and CNS Measles infection. Brain MRI showed symmetrical high signal intensity in basal ganglia bilaterally and periventricular white matter changes (Figure 1).

EEG showed generalized epileptiform discharges with periodic pattern.

Patient was started on IVIG $2 \mathrm{~g} / \mathrm{kg}$ for 5 days followed by a course of pulse steroids, mythylprednisolone of $30 \mathrm{mg} / \mathrm{kg} /$ day for 3 days followed by oral prednisolone of $2 \mathrm{mg} / \mathrm{kg} /$ day with a plan of weaning over 6 weeks. On day 
two of immunoglobuline infusion treatment, patient started to improve and hyperkinetic movements almost disappear at the end of IVIG course.

With continuation of pulse steroid course he started to be aware about his parents, responding to his name, smiling and asking for food. From infectious disease prospective he was started on ribavirin a dose of $10 \mathrm{mg} / \mathrm{kg}$ three times a days for 3 days then twice a day for total of
14 weeks and Cemetidine in a dose of $7 \mathrm{mg} / \mathrm{kg} / \mathrm{dose}$ for 14 weeks. He was on sodium valproate and clonazepam and seizures were controlled. Patient was discharged and returned back to Yemen given appointment in neurology clinic for follow up. Patient was seen in a clinic 2 months later and showed improvement and controlled seizures, myoclonus still present but better in compare before treatment.
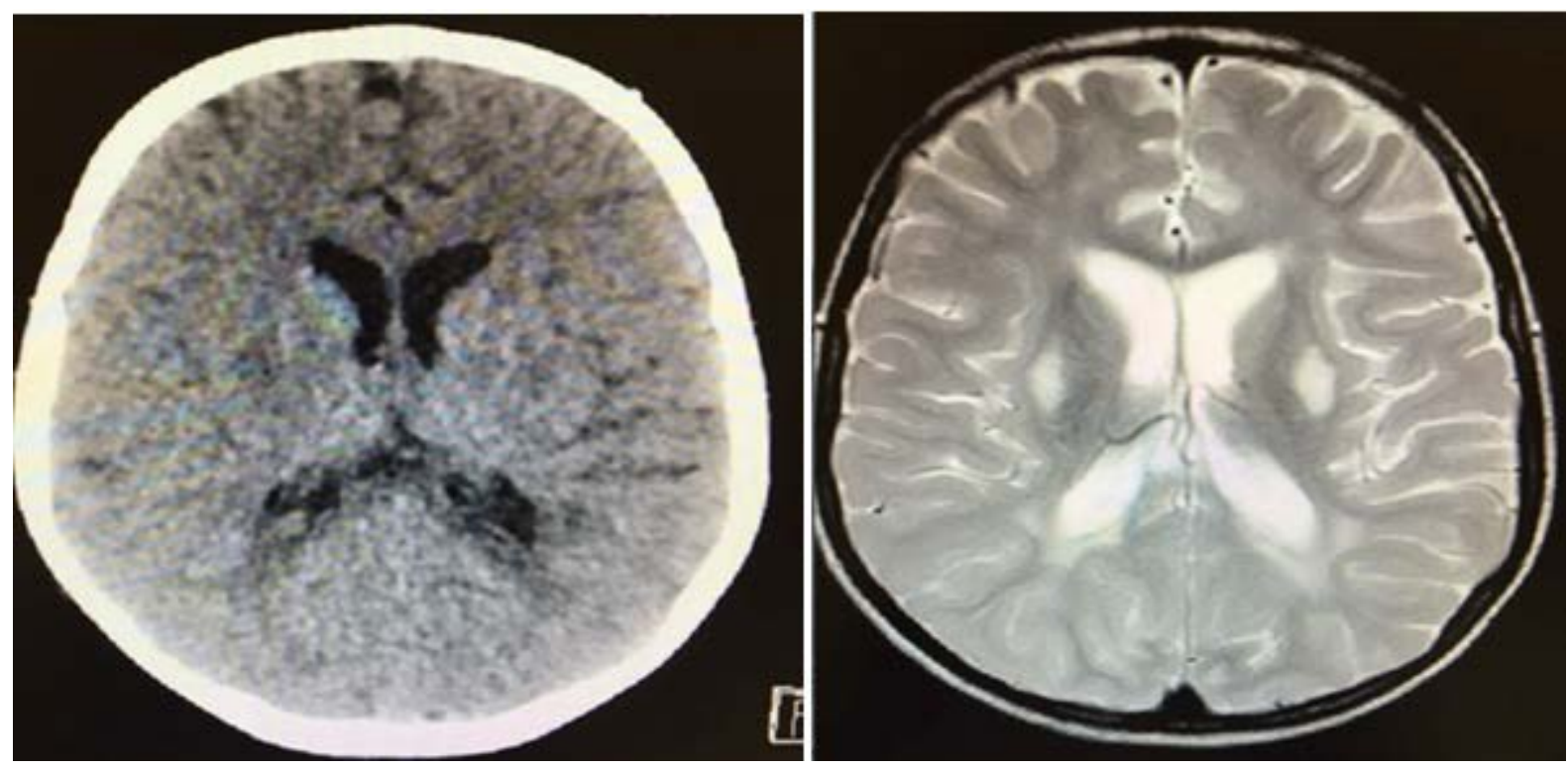

Figure 1. Brain CT, axial section, showed bilateral a symmetrical hyposensitizes in basal ganglia and periventricular white matter changes. Brain MRI, axial, T2 study high signal intensity in basal ganglia bilaterally involving lentiform nucleus and periventricular white matter

\section{Discussion}

Sub-acute sclerosing pan-encephalitis (SSPE) is a rare chronic, persistent encephalitis secondary to a natural measles infection that causes widespread demyelination of the CNS. The clinical features and time course of SSPE are highly variable. The diagnosis is based on characteristic clinical features, periodic electroencephalographic complexes and elevated measles antibody titer in cerebrospinal fluid. After appearance of myoclonus the diagnosis of SSPE can reliably be established with the help of Dyken's diagnostic criteria: (1) A typical clinical picture of progressive subacute mental deterioration with stereotyped generalized myoclonus, (2) Characteristic electroencephalogram changes, (3) Elevated cerebrospinal fluid globulin levels greater than $20 \%$ of total cerebrospinal fluid protein, (4) Raised cerebrospinal fluid measles antibody titers, (5) Typical histopathologic findings in brain biopsy or autopsy.

The pathological process SSPE Measles virus infection of brain tissue is marked by the absence of lytic infection, transcription or translation of structural measles proteins. Only intracellular measles RNA replication is being maintained using the ribonucleoprotein (TNP) complex. Cell to cell spread of RNP complex in the brain using cell-cell fusion events takes place over few years before clinical onset. [4,5] During the slow replication of the RNA, naturally occurring mutation happens at constant rate and allowed to build up in numbers due to the absence of lytic infection that otherwise select the more functionally fit wild strain. Furthermore, there is evident of presence of hypermutation attributed to cellular RNA dependent adenosine deaminase enzyme. This hyper mutation leads to replacement of uridine (U) to cytidine (C) in SSPE genome particularly in the $\mathrm{M}$ region where U-to-C transitions can reach up to $50 \%[4,6]$.

In SSPE, measles RNA is usually not detected in CSF and brain tissue is preferred for measles molecular detection and isolation. This might be due to the intracellular infection of brain tissue without lytic infection. However, measles antibodies will be present in abundance in the CSF.

In our patient all criteria met except of brain biopsy. Regarding treatment, several reports suggested that combination of two or more than two drugs were more effective in stabilizing the course of the disease. Combination therapy appeared to prolong the survival time of patients. $[7,8]$ In our patient we used IVIG and steroids initially followed by ribavirin and Cemetidine. The use of intravenous immunoglobulin in SSPE patients has only been mentioned in a single case report [6]. Report a 10 -year-old boy treated with intravenous immunoglobulin and inosiplex for 18 months [7,8]. The use of intravenous immunoglobulin in SSPE patients has only been mentioned in a single case report [8]. Reported on a 24-month-old boy who developed a rapidly progressing disease course after adrenocorticotropic hormone was added to the therapy for infantile spasms [9]. The authors attributed this to the immunosuppressive effects of glucocorticoid therapy. There is unpublished experience of rapid deterioration in a 9-year-old boy after pulse steroid therapy [10].

In our patient, after a course of IVIG and pulse steroid, patient improved, he can recognize parents and relatives, asking for food, smiling and become more interactive with 
parents, choreathetoid movements completely resolved. Myoclonus present with some improvement, patient started on sodium valproate and clonazepam. Patient also started on ribavirin and Cemetidine but dramatic improvement was noticed with immunoglobulin and pulse steroid. On subsequent follow up, the child still has myoclonus and he was treated with carbamazepine.

\section{Conclusion}

SSPE should be considered in any child who present with progressive motor function and mental changes who came from countries with low measles vaccine coverage. Though with previous reports about variable responsive to immunoglobulin IV and a case report with deterioration in steroids. In our patient there is dramatic improvement with immunoglobulin and steroids.

\section{References}

[1] R.K. Garg B. Karak. A.M. Sharma Subacute Sclerosing Panencephalitis. INDIAN PEDIATRICS. VOLUME 35-APRIL 1998.
[2] Jabbour JT, Garcia JH, Lemmi H, Regland J, Duenas DA, Sever JL. Subacute sclerosing panencephalitis. A multidisciplinary study of eight cases. JAMA 1969; 207: 2248-54.

[3] Prashanth LK,Taly AB, Ravi V, Sinaha S, Rao S. Long term survival in subacute sclerosing panencephalitis: an enigma. Brain Dev 2006; 28: 447-52.

[4] Jurgen Schneider-Schaulies et.al. Measles virus in the CNS: the role of viral and host factors for the establishment and maintenance of a persistent infection. Journal of NeuroVirology 1999; 5, 613-622.

[5] Rosina Giordano-Santini, Casey Linton, Massimo A. Hilliard. Cell-cell fusion in the nervous system: Alternative mechanisms of development, injury, and repair. Seminars in Cell \& Developmental Biology 60 2016; 146-154.

[6] Cattaneo, R.; Schmid, A.; Eschle, D.; Baczko, K.; terMeulen, V.; Billeter, M.A. Biasedhypermutationandother genetic changes in defective measles viruses in human brain infections. Cell 1988; 55, 255-265.

[7] Gürer YKY, Kükner Ş, Sarica B. Intravenous y-globulin treatment in a patient with subacute sclerosing panencephalitis. Pediatr. Neurol. 1996; 14(1), 72-74.

[8] Ravindra Kumar Garg,subacute sclerosing panencephalitis, J Neurol 2008; 255:1861-1871.

[9] Serdaroglu G, Kutlu A, Tekgul H, Tutuncuoglu S. Subacute sclerosing panencephalitis: a case with fulminant course after ACTH. Pediatr. Neurol. 2004; 31(1), 67-70.

[10] Burak Tatlı, Barış Ekici and Meral Özmen, Current therapies and future perspectives in subacute sclerosing panencephalitis, Expert Rev. Neurother. 2012; 12(4), 485-492. 